\title{
Promouvoir les services de vasectomie en Haïti
}

\author{
Brian Perry \\ Catherine Packer \\ Dawn S. Chin-Quee \\ Trinity Zan \\ Dominick Shattuck
}

Follow this and additional works at: https://knowledgecommons.popcouncil.org/departments_sbsr-rh How does access to this work benefit you? Let us know!

\section{Recommended Citation}

Perry, Brian, Catherine Packer, Dawn S. Chin-Quee, Trinity Zan, and Dominick Shattuck. 2016. "Promouvoir les services de vasectomie en Haïti." Durham, NC: FHI 360 and Washington, DC: The Population Council, The Evidence Project. 


\section{PROMOUVOIR LES SERVICES DE VASECTOMIE EN HAÏTI}

Le mouvement de la Planification Familiale 2020 (FP2020) a commencé en 2012, lorsque plus de 150 dirigeants politiques, au Sommet de Londres sur la Planification Familiale (London Summit on Family Planning) se sont engagés à fournir l'accès aux contraceptifs d'ici 2020 à 120 million de femmes des pays les plus pauvres du monde. FP2O20 vise à soutenir les droits reproductifs des femmes et des jeunes filles et leur donner les moyens d'achever leurs objectifs en matière de reproduction. Cela se fait, en partie, en s'assurant que les clientes de la PF à travers le monde reçoivent avec le choix éclairé, un large éventail de méthodes contraceptives de haute qualité. Relatif à FP2020, le gouvernement d'Haïti s'est engagé à repositionner la PF comme une priorité nationale et augmenter le taux de prévalence contraceptive parmi les femmes mariées à 50\% d'ici 2016, et de réduire les besoins non satisfaits de la PF en améliorant la sécurité des produits de base. ${ }^{1}$ L'extension du mélange de méthodes grâce à renforcer la capacité, encourager la demande pour la vasectomie, et créer les services $\mathrm{PF}$ « pour les hommes » augmente la prévalence contraceptive moderne $^{2}$ et aide Haïti à progresser vers ses objectifs de PF.

Cependant, la gamme actuelle de méthodes de PF accessibles, les messages de changement de comportement et la communication interpersonnelle renforcent l'idée que les services de PF sont spécifiquement pour les femmes. Les idées fausses au sujet de la vasectomie, limitent le soutien financier, les politiques et le soutien communautaire, pour la méthode. A ce jour, seulement quelques petits programmes privés se sont efforcés d'accroître la promotion et de fournir la vasectomie sans bistouri en Haïti. En conséquence, les hommes sont insuffisamment engagés dans les services de santé de la reproduction et les femmes portent la responsabilité principale d'utiliser la contraception.

La vérité est que les services de vasectomie apportent aux couples cherchant à limiter la taille de leur famille, une alternative de PF très efficace, de nature permanente, et axée sur l'homme, sans effets secondaires.

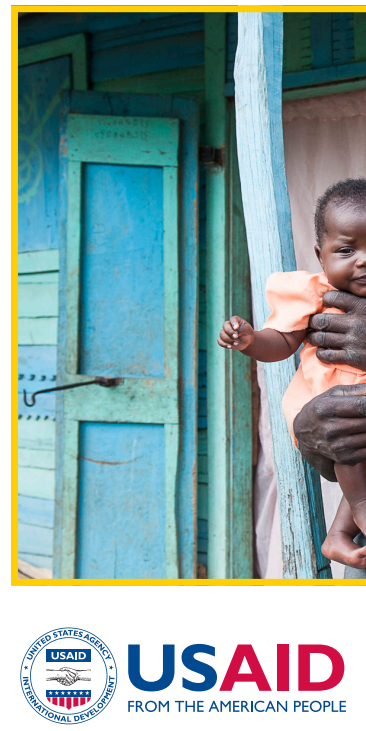

La vasectomie sans bistouri:

- $\quad$ Est SANS DOULEUR, RAPIDE, et EXTRÊMEMENT EFFICACE

- Exige PEU DE RÉCUPÉRATION,

- Apporte DES AVANTAgES ÉCONOMIQUES ET SANITAIRES à la famille,

- N'A PAS D'EFFETS SECONDAIRES, et

- N'AFFECTE EN RIEN LA FONCTION SEXUELLE OU LA JOUISSANCE.

Une plus grande éducation et le soutien à la vasectomie au niveau national traiterait le déséquilibre entre les sexes dans la disponibilité et l'utilisation des contraceptifs. Contrairement à une nouvelle méthode concentrée sur la femme, l'intégration significative de la vasectomie dans un mélange de méthodes, élargit le paradigme de la santé de la reproduction pour inclure les hommes en tant qu'utilisateurs des services de la PF et promouvoir la fertilité des couples comme une responsabilité partagée. Il existe certaines preuves anecdotales sur la demande de vasectomie basées sur "les voyages de mission sur la vasectomie" menés par des urologues des États-Unis qui ont eu une forte participation de clients. Cette approche plus holistique envers la santé de la reproduction soutient un choix éclairé d'un large éventail de méthodes contraceptives de haute qualité.

La vasectomie est la méthode permanente la plus rentable et la plus sûre pour répondre aux besoins des hommes et des femmes qui souhaitent limiter les naissances (ne veulent plus d'enfants). Vu l'engagement d'Haïti à prioriser la PF et élargir l'accès et l'utilisation de méthodes modernes, maintenant, est le moment idéal d'investir dans les services de vasectomie. En favorisant et en soutenant l'utilisation volontaire de la vasectomie dans le cadre global du mélange de méthodes, Haïti peut répondre à ses engagements nationaux et internationaux en matière de PF et contribuer au développement d'une société plus équitable en matière de genre, où les hommes jouent un rôle proactif et de soutien dans la santé de leurs familles.

Une plus grande intégration de la promotion et des services de vasectomie est une première étape dans l'expansion du paradigme de la santé de la reproduction pour inclure les hommes et pas seulement en tant que partenaires par défaut des clientes, mais en tant que bénéficiaire égal des programmes de santé de la reproduction. 


\section{LA VASECTOMIE AIDE LES PARTICULIERS ET LES COUPLES À ACHEVER LA TAILLE DÉSIRÉE DE LEUR FAMILLE}

En Haïti, 53\% des hommes mariés ${ }^{3}$ et $58 \%$ des femmes mariées ${ }^{4}$ en âge de procréer ont signalé un souhait de limiter les futures naissances. Les hommes et les femmes plus âgés étaient beaucoup plus susceptibles de vouloir limiter les naissances ${ }^{3,4}$ ( $92 \%$ de ces hommes et $75 \%$ de ces femmes étaient âgés d'au moins 30 ans) même si un nombre important de personnes plus jeunes sont aussi intéressées à limiter la taille de la famille.

Estimation de la taille du marché pour les méthodes permanentes en Haïti, $2020^{3,4,5}$

\begin{tabular}{|c|c|c|c|}
\hline Population totale des femmes & $5,383,035$ & $5,309,691$ & Population totale des hommes \\
\hline Mariées (âgées de 15 à 49 ans) & 546,690 & 625,751 & Mariés (âgés de 20 à 59 ans) \\
\hline Désir de limiter les futures naissances* & 347,420 & 356,606 & e limiter les futures naissances* \\
\hline
\end{tabular}

*Exclut les hommes et les femmes qui déclarent une stérilisation antérieure ou l'infertilité

\section{LA VASECTOMIE ÉLARGIT LE CHOIX DES MÉTHODES EFFICACES PERMANENTES DE CONTRACEPTION}

En Haïti, 94\% des femmes mariées qui ont déclaré un désir de limiter les futures naissances utilisaient soit des méthodes contraceptives à court terme ou n'utilisaient aucune méthode. ${ }^{4}$ Les injectables, qui ont un taux élevé d'abandon en raison d'effets secondaires, représentent plus de 59\% des contraceptifs utilisés par cette population. La vasectomie offre une durée de vie de la protection contraceptive sans soucis des effets secondaires.
Utilisation de la contraception chez les femmes mariées (15 à49 ans) qui veulent limiter de futures naissances en $\mathrm{Haïti}^{4}$
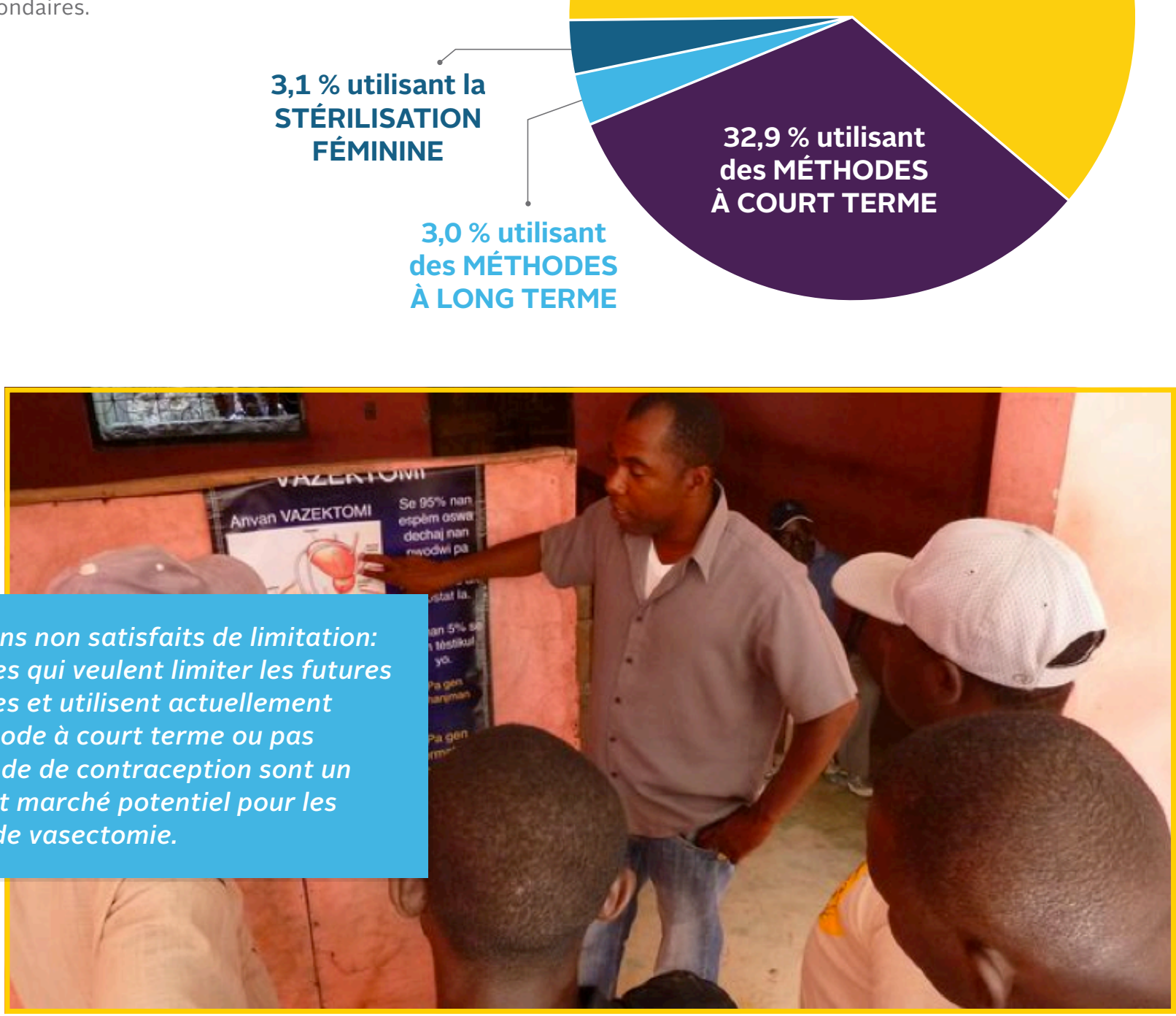

Les besoins non satisfaits de limitation: les couples qui veulent limiter les futures naissances et utilisent actuellement une méthode à court terme ou pas de méthode de contraception sont un important marché potentiel pour les services de vasectomie. 


\section{L’UTILISATION PLUS ÉLEVÉE DE VASECTOMIE RÉDUIT} LES TAUX DE GROSSESSES NON DÉSIRÉES ET LES COÛTS GLOBAUX DE SERVICES DE SANTÉ REPRODUCTIVE

Si Haïti devait atteindre son objectif d'augmenter la prévalence contraceptive à 50\% parmi les femmes mariées (âgées de 15 à 49 ans) d'ici 2016 et l'utilisation des contraceptifs continuaient d'augmenter à la même vitesse, il y aurait environ 352.000 utilisateurs de contraceptifs dans ce groupe démographique d'ici 2020. Si un faible pourcentage de besoins non satisfaits pour la limitation des couples mariés en Haïti a été satisfait grâce à une meilleure absorption de la vasectomie, cela aurait un impact direct et durable sur la santé publique et réduirait les coûts globaux des produits et des services de PF.

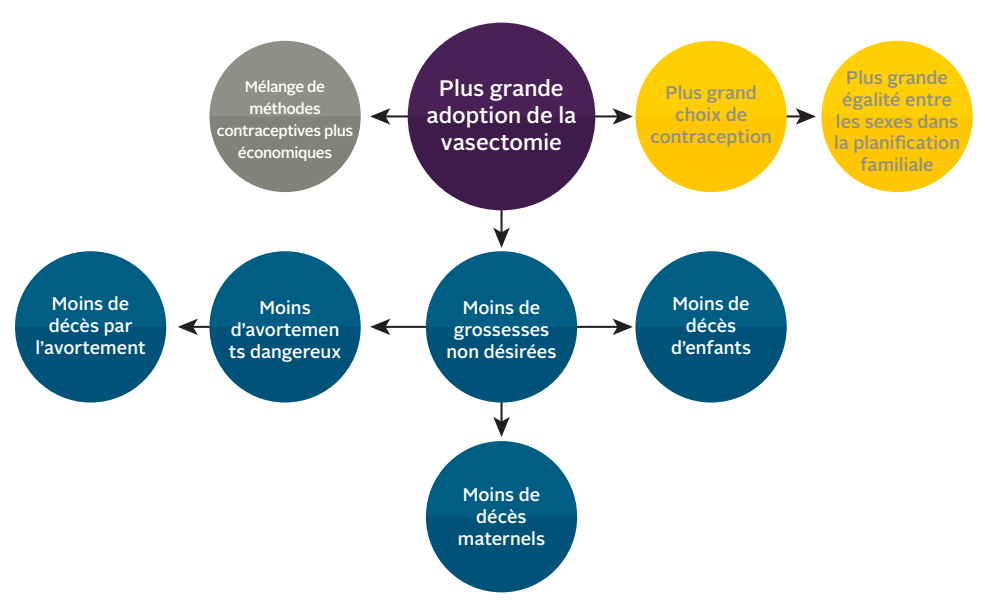

\section{LA VASECTOMIE EST PARMI LES MÉTHODES DE PLANIFICATION FAMILIALE LES PLUS RENTABLES}

La vasectomie coûte moins a que toutes autres formes de contraceptifs actuellement fournis par années de protection pour un couple, à l'exception des DIU au cuivre. ${ }^{6,7}$ En Haïti, un investissement accru dans la vasectomie entrainerait plus d'économies par années de protection pour un couple en coûts de produits et service de la méthode de mélange contraceptive de la nation.

Coût de fourniture de contraceptifs par années de protection pour un couple au Burundi, 2015

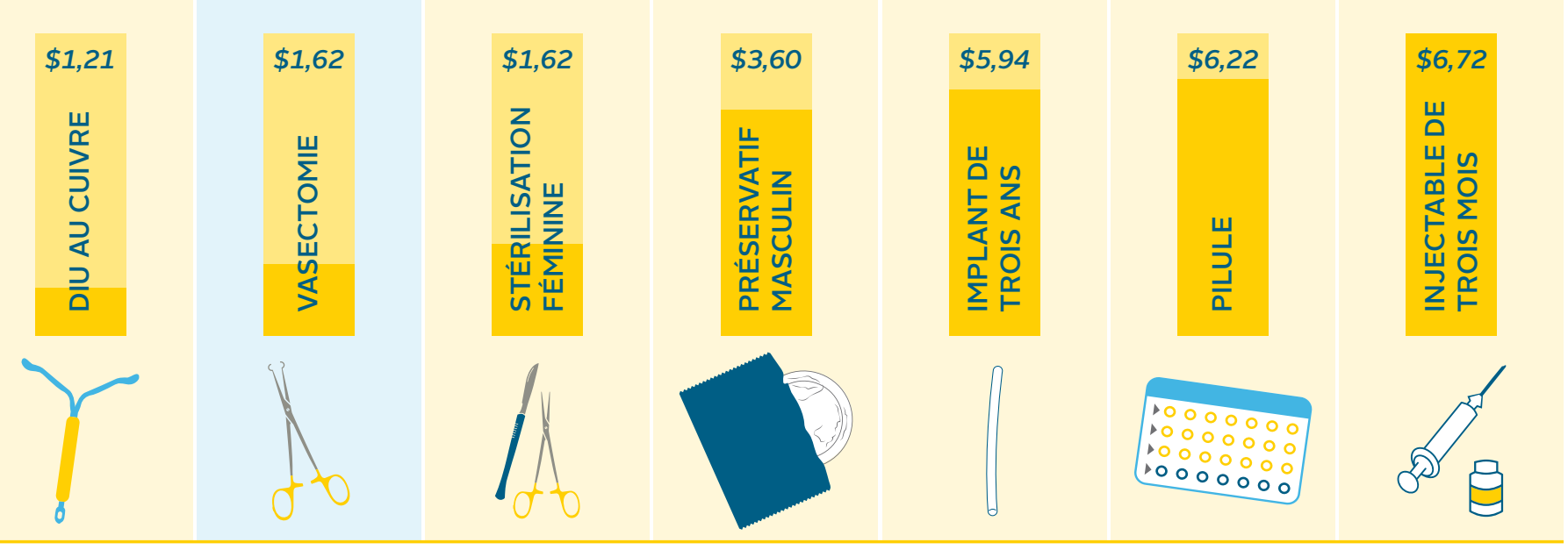

Les coûts par années de protection pour un couple ne reflètent que la main-d'oeuvre directe et le coût des matières premières et des fournitures consommables et ne prend pas en compte d'autres coûts potentiels associés à l'introduction de la vasectomie sur le marché, y compris la création de la demande, les formations de prestataire supplémentaires ou l'expansion de la couverture des services.

\section{LA VASECTOMIE PEUT CONTRIBUER À UNE COMBINAISON DE MÉTHODES MOINS COÛTEUSES ET PLUS EFFICACES}

Par exemple, si d'ici 2020, seulement 5\% des femmes mariées (âgées de 30 à 49 ans) qui souhaitent limiter les naissances passaient de l'utilisation de méthodes à court terme comptant sur la vasectomie de leur partenaire pour achever leurs objectifs de fécondité, cela permettrait d'améliorer le rapport coût-efficacité du mélange de méthodes pour ce groupe démographique par plus de $22 \%$.

Cette augmentation marginale de l'utilisation de la vasectomie pour limiter les naissances - égale à 19.365 des vasectomies réalisées au cours des 5 prochaines années - permettrait d'améliorer l'impact du mélange de méthodes sur la santé publique par plus de $30 \%$.

\section{CUMULATIF COUT-EFFICACITE ET IMPACTS SUR LA SANTE PUBLIQUE $(2016-2020)^{\mathrm{b}}$}

\begin{tabular}{|r|c|c|}
\hline & $\begin{array}{c}\text { Combinaison } \\
\text { de méthodes } \\
\text { actuelle* }\end{array}$ & $\begin{array}{c}\text { Combinaison de } \\
\text { méthodes* dont 5\% de } \\
\text { vasectomie prévalence } \\
\text { contraceptive }\end{array}$ \\
\hline $\begin{array}{r}\text { Coût par années de } \\
\text { protection pour un couple }\end{array}$ & $\$ 5,75$ & $\$ 4,92$ \\
\hline $\begin{array}{r}\text { Coût total par années de } \\
\text { protection pour un couple }\end{array}$ & 577.345 & 682.013 \\
\hline Grossesses non désirées évitées & 166.275 & 196.420 \\
\hline Décès maternels évités & 582 & 687 \\
\hline Décès infantiles évités & 9.810 & 11.589 \\
\hline Avortements dangereux évités & 27.535 & 32.527 \\
\hline Cas de décès à l'avortement évités & 22 & 26 \\
\hline
\end{tabular}

* Parmi les femmes mariées (30 à 49 ans) qui souhaitent limiter les naissances 
Les recommandations ci-dessous, adaptées à partir d'un examen du récent document ${ }^{8,9}$ peuvent aider les décideurs et les exécutants de programme en Haïti à accroître la demande pour la vasectomie, augmenter l'offre de services de vasectomie, et créer un environnement plus propice à un programme de PF incluant les hommes.

\section{CRÉER UN ENVIRONNEMENT FAVORABLE :}

- Mettre en place des politiques de soutien et un appui politique pour l'inclusion des hommes et élaborer un plan de mise en oeuvre budgétisée pour la PF qui comprend l'expansion des services de vasectomie et exige des activités de création.

- Combler l'écart entre les engagements de PF et le soutien financier disponible, les donateurs et le gouvernement devraient investir dans la combinaison de méthodes la plus rentable pour répondre aux besoins changeant des hommes, des femmes, et des couples sur la durée de leur vie reproductive.

- Adresser les normes liées au genre qui peuvent avoir un impact négatif sur la prise de décision en PF, par l'autonomisation des femmes et des couples pour parler ouvertement de leurs intentions en matière de reproduction et considérer la vasectomie comme une façon désirable d'assurer la santé et le bien-être de la famille.

- Inclure la vasectomie dans l'éducation des jeunes sur les méthodes contraceptives, en particulier les jeunes gens, pour commencer la sensibilisation très tôt sur la vasectomie comme une forme viable de la limitation des naissances à l'avenir.

- Créer des services de santé de la reproduction "pour les hommes" en formant des agents de santé du sexe masculin pour conseiller les hommes au sujet de leurs options de PF.

\section{ENCOURAGER LA DEMANDE POUR LA VASECTOMIE:}

- Renforcer des capacités dans le secteur public aux endroits avec des installations d'enseignement médical et les services du secteur privé disponibles, et assez de demande des services pour soutenir le développement des compétences.

- Régler d'éventuels préjugés et les attitudes des prestataires liées à la prestation de services de vasectomie par la formation et les témoignages, et assurer que les prestataires reçoivent une compensation adéquate pour les services.

- Renforcer les capacités des hôpitaux publics et des unités mobiles à offrir la vasectomie sans bistouri avec l'utilisation de l'interposition aponévrotique et la cautérisation thermique dans le cadre de leurs services existants
- Investir dans le développement durable de compétences en vasectomie en formant des prestataires formateurs certifiés dans le secteur public comme formateurs de vasectomie.

\section{ENCOURAGER LA DEMANDE POUR LA VASECTOMIE:}

- Engager et soutenir les "champions" de la vasectomie parmi les leaders religieux, politiques et communautaires, les prestataires de la santé, les agents de santé communautaires, et les clients satisfaits de la vasectomie.

- Diffuser des informations précises sur la vasectomie; en particulier en matière de sécurité de la méthode et son effet sur la virilité et la force physique.

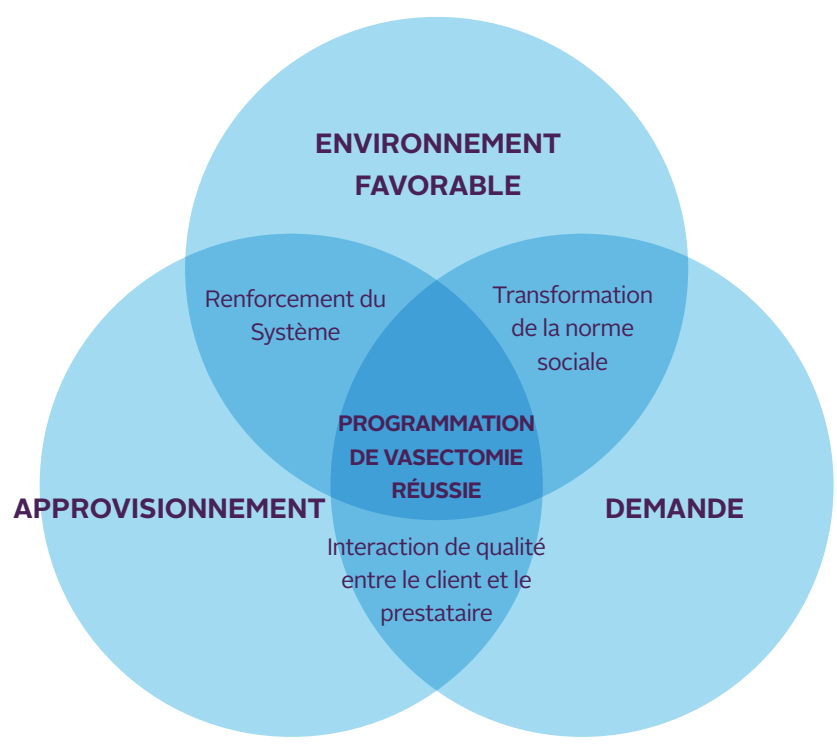

Adapté du Supply-Enabling Environment-Demand (SEED) ProgrammingModel ${ }^{\mathrm{TM}} \mathrm{d}^{\prime}$ EngenderHealth

Ministry of public health and population. Strategic national plan of reproductive health and family planning, 2013-2016. 2012. Disponible à:

http://mspp.gouv.ht/site/downloads/plan\%20intermediaire\%20SR\%20vers\%20def\%20compressed.pdf

Ross J and Stover J. Use of modern contraception increases when more methods become available: analysis of evidence from 1982-2009. Glob Health Sci Pract. 2013;1(2):203-212. Institut Haïtien de l'Enfance [Haïti] and ORC Macro. 2001. Enquête Mortalité, Morbidité et Utilisation des Services 2000. HTMR42FL.SAV. Pétionville, Haïti et Calverton, Maryland: Institut Haitien de l'Enfance and ORC Macro [Producteurs]. ORC Macro [Distributeur], 2001.

Institut Haïtien de l'Enfance [Haïti] and ICF International. 2013. Enquête Mortalité, Morbidité et Utilisation des Services 2012. HTIR61FL.SAV. Pétion-Ville, Haïti et Calverton, Maryland: Institut Haitien de l'Enfance and ICF International [Producteurs]. ICF International [Distributeur], 2013.

US Census Bureau. International Database. Dernière mise à jour juillet 2015. Disponible à: http://www.census.gov/population/international/data/idb/informationGateway.php.

Tumlinson K, Steiner MJ, Rademacher KH, Olawo A, Solomon M, and Bratt J. 2011. The promise of affordable implants: is cost recovery possible in Kenya. Contraception, 83:88-93. Rademacher KH, Solomon M, Pascual C, Njunguru J, Brett T, and Steiner MJ. 2016. Expanding access to a new, more affordable levonorgestrel intrauterine system in Kenya: Perspectives from Key Opinion Leaders. Global Health: Science and Practice. In press.

8 Perry B, Packer C, Chin Quee D, Zan T, Dulli L, and Shattuck D. Recent experience and lessons learned in vasectomy programming in low-resource settings: a document review. Durham, NC: FHI 360 et Washington, DC: Population Council, the Evidence Project. 2016.

Packer C, Perry B, Chin-Quee D, Zan T, and Shattuck D. How to create successful vasectomy programs. Durham, NC: FHI 360 and Washington, DC: The Population Council , the Evidence Project. 2016. 


\section{LE PROJET EVIDENCE}

POPULATION COUNCIL

4301 Connecticut Avenue, NW, Suite 280

Washington, DC 20008 USA

Tel +1202 2379400

Evidenceproject.popcouncil.org

Ce travail est rendu possible grâce au soutien généreux du peuple américain à travers

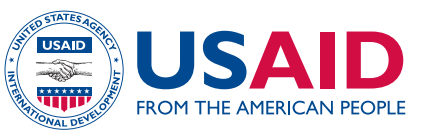

l'Agence Américaine pour le Développement International (USAID) aux termes d'un accord de coopération no. AID-OAA-A-13-00087 du Projet Evidence. Les résultats et les conclusions sont la seule responsabilité des auteurs et ne reflètent pas nécessairement les vues de I'USAID ou du gouvernement des États-Unis.

Le Projet Evidence vise à élargir l'accès aux services de haute qualité de planification

\section{Evidence} familiale/santé reproductive dans le monde entier grâce à la science de la mise en oeuvre, y compris la génération stratégique, la traduction, et l'utilisation de preuves nouvelles et existant. Le projet est dirigé par Population Council en partenariat avec le Réseau INDEPTH, I'International Planned Parenthood Federation, PATH, et le Population Reference Bureau.

\section{fhi 360}

FHI 360 est une organisation de développement humain sans but lucratif vouée à l'amélioration de la vie de façon durable en avançant des solutions intégrées, dirigées localement. Notre personnel comprend des experts en santé, en éducation, nutrition, environnement, développement économique, la société civile, le sexe, les jeunes, la recherche et la technologie - créant un mélange unique de capacités pour relever les défis de développement interdépendants d'aujourd'hui. FHI 360 sert plus de 60 pays, les 50 états américains et tous les territoires américains.

Pour plus d'informations sur les recommandations programmatiques sur la vasectomie, veuillez contacter:

\section{FHI 360}

Mailing address:

359 Blackwell Street,

Suite 200, Durham, NC 27701

Téléphone: +1 9195447040

Fax: +1 9195447261

Publié en Mars 2016

Citation Suggéré:

Perry B, Packer C, Chin-Quee D, Zan T, and Shattuck D. Promoting Vasectomy Services in Kenya. Durham, NC: FHI 360 and Washington, DC: The Population Council, the Evidence Project. 2016.

Copyright (c) 2016 FHI 360. Tous droits réservés. 


\section{PHOTO CITATIONS}

\section{Photo 1 - ID: 2602-22}

Simeus tient la plus jeune de ses quatre filles au Plateau Central, Haïti.

(c) 2014 C. Hanna-Truscott/Midwives for Haïti, avec l'autorisation de Photoshare

\section{Photo 2}

Des hommes se sont réunis autour d'une affiche pour se parler.

(c) Doug Stein/NSVI 